Clinical Research

\title{
Colchicine's effects on metabolic and inflammatory molecules in adults with obesity and metabolic syndrome: results from a pilot randomized controlled trial
}

\author{
Andrew P. Demidowich $\mathbb{1}^{1,2,3,5} \cdot$ Jordan A. Levine ${ }^{1} \cdot$ Richard Apps $^{4} \cdot$ Foo K. Cheung ${ }^{4} \cdot$ Jinguo Chen $^{4}$. \\ Giovanna Fantoni $^{4} \cdot \mathrm{CHI}$ Consortium $\cdot$ Tushar P. Patel $^{1} \cdot$ Jack A. Yanovski $^{1}$
}

Received: 23 March 2020 / Revised: 3 May 2020 / Accepted: 7 May 2020 / Published online: 27 May 2020

(c) US GOVT 2020

\begin{abstract}
Objective Recent clinical trials have demonstrated that colchicine may have metabolic and cardiovascular and benefits in atrisk patients; however, the mechanisms through which colchicine may improve outcomes are still unclear. We sought to examine colchicine's effects on circulating inflammatory and metabolic molecules in adults with obesity and metabolic syndrome (MetS).

Methods Blood samples were collected pre- and post-intervention during a double-blind randomized controlled trial in which 40 adults with obesity and MetS were randomized to colchicine $0.6 \mathrm{mg}$ or placebo twice-daily for $3 \mathrm{months}$. Serum samples were analyzed for 1305 circulating factors using the SomaScan Platform. The Benjamini-Hochberg procedure was used to adjust the false discovery rate (FDR) for multiple testing.

Results At baseline, age $(48.0 \pm 13.8$ vs. $44.7 \pm 10.3$ years $)$ and BMI $\left(39.8 \pm 6.4 \mathrm{vs} .41 .8 \pm 8.2 \mathrm{~kg} / \mathrm{m}^{2}\right)$ were not different between groups. After controlling for the FDR, 34 molecules were significantly changed by colchicine. Colchicine decreased concentrations of multiple inflammatory molecules, including C-reactive protein, interleukin 6, and resistin, in addition to vascular-related proteins (e.g., oxidized low-density lipoprotein receptor, phosphodiesterase 5A). Conversely, relative to placebo, colchicine significantly increased concentrations of eight molecules including secreted factors associated with metabolism and anti-thrombosis.

Conclusions In adults with obesity, colchicine significantly affected concentrations of proteins involved in the innate immune system, endothelial function and atherosclerosis, uncovering new mechanisms behind its cardiometabolic effects. Further research is warranted to investigate whether colchicine's IL-6 suppressive effects may be beneficial in COVID-19.
\end{abstract}

Members of the CHI Consortium are listed below Acknowledgements.

Supplementary information The online version of this article (https:// doi.org/10.1038/s41366-020-0598-3) contains supplementary material, which is available to authorized users.

Andrew P. Demidowich

ademido1@jh.edu

1 Section on Growth and Obesity, Division of Intramural Research, Eunice Kennedy Shriver National Institute of Child Health and Human Development (NICHD), National Institutes of Health (NIH), Bethesda, MD 20892-1103, USA

2 Office of the Clinical Director, NICHD, NIH, Bethesda, MD 20892, USA

\section{Introduction}

Individuals with obesity and metabolic syndrome (MetS) have an increased risk for Type 2 Diabetes (T2D) and cardiovascular disease (CVD) development and premature death [1]. Having chronic inflammation adds further risk of developing CVD or an acute cardiac event [2,3]. Given that worldwide an estimated $25 \%$ of adults have MetS, it is

3 Department of Endocrinology, Diabetes and Metabolism, Johns Hopkins School of Medicine, Baltimore, MD 21205, USA

4 Center for Human Immunology (CHI), National Institute of Allergy and Infectious Diseases, NIH, Bethesda, MD 20892, USA

5 Present address: Johns Hopkins Community Physicians at Howard County General Hospital, Johns Hopkins Medicine, Columbia, MD 21044, USA 
clinically important to identify potential treatments for MetS to prevent development of serious comorbid conditions [4].

Colchicine, an anti-inflammatory medication used for gout, has garnered considerable interest for its potential metabolic and cardiovascular benefits. Colchicine can reduce fasting insulin resistance in adults with obesity and has been suggested to reduce the risk of incident T2D $[5,6]$. Among individuals with CVD, colchicine also decreased the incidence of major adverse cardiovascular events [7].

The mechanism through which colchicine exerts its beneficial metabolic and cardioprotective effects has been attributed to its anti-inflammatory actions, both systemically and in local tissues [5, 8]. Indeed, a pilot randomized controlled trial (RCT) of colchicine's effects that we carried out in adults with obesity, MetS, and inflammation found that a measure of insulin resistance, HOMA-IR, as well as the inflammatory markers $\mathrm{C}$-reactive protein (CRP), erythrocyte sedimentation rate (ESR), and GlycA were all significantly reduced in the colchicine versus the placebo group [5, 9]. However, a comprehensive investigation of the inflammatory pathways affected by colchicine in obesity has not been performed. Moreover, how changes in specific inflammatory mediators relate to colchicine's effects on metabolic biomarkers, such as insulin resistance or insulin secretion, are also not well understood. As microtubules are ubiquitously expressed and are integral for many cellular processes, it is plausible that colchicine exerts salutary cardiometabolic effects via noninflammatory pathways as well.

The aim of this secondary analysis of our prior RCT is to evaluate colchicine's effects on circulating metabolic and inflammatory mediators in adults with obesity and MetS. A secondary aim is to better understand the potential pathways by which colchicine improves fasting insulin resistance in obesity [5].

\section{Materials and methods}

\section{Study design}

The details and methods of this single-center, double-blind, randomized, placebo-controlled trial have been described previously [5]. Subjects were randomized in a 1:1 ratio to receive colchicine (Spectrum Chemical Corp, New Brunswick, NJ) or identically appearing placebo capsules twicedaily for 3 months. The primary outcome was change in insulin sensitivity. The study protocol was approved by the Eunice Kennedy Shriver National Institute of Child Health and Human Development Institutional Review Board, and participants provided written consent for participation. No participant, investigator, or other staff interacting with participants was aware of study group assignment during the trial.

\section{Participants}

A convenience sample of 40 adults with obesity (BMI $\geq$ $30 \mathrm{~kg} / \mathrm{m}^{2}$ ) and MetS were studied at the NIH Clinical Research Center between 2014 and 2018. At baseline, participants were required to have evidence for chronic inflammation (hsCRP $\geq 19.0 \mathrm{nmol} / \mathrm{L})$ and insulin resistance (Homeostatic model of insulin resistance [HOMAIR] $\geq 2.6$ ). Subjects with significant chronic medical conditions including diabetes mellitus, CVD or taking medications affecting glucose homeostasis (e.g., metformin and insulin), lipids/cholesterol (e.g., statins and fibrates), body weight, or inflammation (e.g., steroids and NSAIDs) were excluded. Exclusion criteria for premenopausal female participants included irregular menses, pregnancy, breastfeeding, planning pregnancy in the next 6 months, or being unwilling to use contraception during their participation in the study.

\section{Laboratory measurements}

Blood samples were drawn at baseline and after 3 months of study drug. Peripheral blood was collected after overnight fasting in serum tubes and centrifuged for $10 \mathrm{~min}$ at $3500 \mathrm{rpm}$. Obtained serum was immediately stored at $-80^{\circ} \mathrm{C}$ until further analysis without being exposed to freeze-thaw cycles, according to NIH Center for Human Immunology protocols (https://chi.niaid.nih.gov/web/new/ our-research/sop.html). Serum proteomic analysis used the SOMAscan 1.3k Assay (SomaLogic, Boulder, CO). This aptamer-based assay can detect 1305 protein analytes in human serum [10]. The proteins quantified include cytokines, hormones, growth factors, receptors, kinases, proteases, protease inhibitors, and structural proteins. A complete list of analytes measured can be found at http:// somalogic.com/wp-content/uploads/2017/06/SSM-045-

Rev-2-SOMAscan-Assay-1.3k-Content.pdf. Concentrations are measured as relative fluorescence units. The assay was performed according to manufacturer specifications, with data inspection using a web tool, as previously described [11]. First-phase insulin secretion (AIRg) was calculated from the first $14 \mathrm{~min}$ of an insulin-modified 3-h frequently sampled intravenous glucose tolerance test, and insulinindependent glucose disposal $\left(S_{\mathrm{G}}\right)$ was calculated using minimal model analysis (SAAM II, The Epsilon Group, Charlotte, VA) as described previously [5]. Oxidized LDL (U/L) concentrations were determined by anti-ApoB-100 conformational epitope 4E6 antibody (Mercodia, Uppsala, Sweden, CAT\# 10-1143-01) [9]. Whole-body fat mass and fat-free mass were obtained by dual-energy $\mathrm{x}$-ray 
absorptiometry (GE Lunar iDXA, GE Healthcare, Madison, WI; software GE enCore 15 with CoreScan algorithm) [5].

\section{Statistical analysis}

This was a secondary analysis assessing for differences between treatment arms using Student's $t$ test for normally distributed data or Mann-Whitney $U$ test for nonnormally distributed data. Pearson and Spearman correlation were used for normally distributed and nonnormally distributed data, respectively. Correlation analyses were restricted to proteins significantly affected by colchicine, after adjustment for false discovery rate (FDR). Two-sided significance tests were performed for all analyses. The Benjamini-Hochberg procedure was used to adjust for the FDR for multiple testing. SPSS v25.0 (IBM Corp, Armonk, NY) was used for statistical analyses.

\section{Results}

Pre- and post-intervention samples from 35 subjects (colchicine $n=17$, placebo $n=18$ ) were available for testing. Baseline characteristics were similar between colchicinetreated and placebo-treated groups [Table 1]. Colchicine use did not significantly affect weight $(-0.1 \pm 2.3$ vs. $1.4 \pm$ $2.9 \mathrm{~kg} ; p=0.45)$ or fat-free mass $(0.1 \pm 1.6$ vs. $0.7 \pm 1.5 \mathrm{~kg}$; $p=0.46$ ). Using the nominal significance threshold of $p<0.05$, concentrations of 75 molecules increased and 101 molecules decreased in the colchicine arm as compared with

Table 1 Baseline participant characteristics.

\begin{tabular}{lll}
\hline Variable & Colchicine $(n=17)$ & Placebo $(n=18)$ \\
\hline Age $(\mathrm{y})$ & $48.0 \pm 13.8$ & $44.7 \pm 10.3$ \\
Race $(n, \%)$ & \\
Black & $5,29 \%$ & $4,22 \%$ \\
Non-Black & $12,71 \%$ & $14,78 \%$ \\
Sex $(n, \%)$ & & \\
Female & $12,71 \%$ & $14,78 \%$ \\
Premenopausal female & $4,24 \%$ & $6,33 \%$ \\
Weight $(\mathrm{kg})$ & $113.2 \pm 22.1$ & $118.8 \pm 31.8$ \\
Body mass index $\left(\mathrm{kg} / \mathrm{m}^{2}\right)$ & $39.8 \pm 6.4$ & $41.8 \pm 8.2$ \\
Body fat $(\%)$ & $48.1 \pm 4.1 \%$ & $48.6 \pm 5.8 \%$ \\
Fat-free mass $(\mathrm{kg})$ & $59.5 \pm 12.0$ & $61.5 \pm 13.5$ \\
Fasting glucose $(\mathrm{mg} / \mathrm{dL})$ & $106.2 \pm 10.2$ & $100.8 \pm 7.3$ \\
HOMA-IR & $6.9 \pm 2.9$ & $6.1 \pm 2.7$ \\
hsCRP (mg/L) & $7.8 \pm 7.9$ & $6.5 \pm 4.2$ \\
\hline
\end{tabular}

There were no significant demographic or anthropometric differences between groups at baseline. Data shown as mean $\pm \mathrm{SD}$, except where otherwise noted.

HOMA-IR homeostatic model of insulin resistance, hsCRP highsensitivity C-reactive protein. placebo [Supplementary Table 1]. After controlling for the FDR, 34 molecules remained significantly changed by colchicine [Table 2].

As expected, multiple inflammatory molecules were decreased by colchicine [Fig. 1]. Among them, seven have specific roles with regards to neutrophil function: alpha 1antichemotrypsin, bactericidal/permeability-increasing protein, CD177, matrix metalloproteinase 9 (MMP9), myeloperoxidase, proteinase 3, and S100A12. Other mediators of the innate immune system were also suppressed, including complement components $\mathrm{C} 5 \mathrm{a}$ and $\mathrm{C} 9$, cyclooxygenase-2, haptoglobin, serum amyloid $\mathrm{P}$, and surfactant protein $\mathrm{D}$. In addition, oxidized LDL receptor (LOX-1) and phosphodiesterase 5A concentrations were decreased in the colchicine arm. Conversely, colchicine significantly increased several molecules involved in metabolism and tissue repair (growth/differentiation factor 15 [GDF15], heart-type fatty acid binding protein $[\mathrm{hFABP}]$, hepatocyte growth factor activator) as well as an anti-thrombotic molecule, protein C.

Among colchicine-treated subjects, decreases in fasting glucose were most highly correlated with hFABP $(\rho=$ $-0.61, p=0.009)$ and decreases in fasting insulin resistance, as measured by HOMA-IR, were most highly correlated with GDF15 $(\rho=-0.63, p=0.006)$. Changes in first-phase insulin secretion were most highly correlated with IL-6 $(\rho=0.63, p=0.009)$ and protein $\mathrm{C}(r=-0.63$, $p=0.009$ ), while changes in $S_{\mathrm{G}}$ were most highly correlated with karyopherin alpha $2(r=0.66, p=0.005)$ and IL-6 $(\rho=0.64, p=0.008)$. These correlations were all nominally significant, but did not survive FDR. Changes in LOX-1 were not significantly correlated with changes in oxidized LDL $(r=-0.19, p=0.46)$.

\section{Discussion}

Previous studies have examined colchicine's clinical and inflammatory effects in secondary or tertiary prevention, after patients developed significant CVD [7]. Among individuals with obesity prior to development of significant comorbidity, a pilot RCT found colchicine reduced CRP and ESR, improved fasting insulin resistance, with a trend towards improvement of fasting glucose, fasting insulin and insulin-independent glucose disposal $\left(S_{\mathrm{G}}\right)$ [5]. However, colchicine's effects on different inflammatory pathways in obesity, and how these pathways are associated with colchicine's effects on metabolic parameters, are largely unknown.

In the present study, we found that colchicine affected multiple important circulating metabolic and inflammatory mediators in individuals with obesity and MetS. Specifically, GDF15, a growth factor associated with diabetes and CVD, was increased by colchicine in our study. Decreases 
Table 2 Biomarkers significantly affected by colchicine after controlling for false discovery rate.

\begin{tabular}{|c|c|c|c|}
\hline Pathway/system & Biomarker & $\begin{array}{l}\text { Change for colchicine vs. placebo } \\
(95 \% \text { CI })\end{array}$ & Nominal $p$ value \\
\hline \multicolumn{4}{|l|}{ Decreased } \\
\hline \multirow[t]{20}{*}{ Inflammatory } & $\mathrm{A} 1 \mathrm{AC}$ & $-29.6 \%(-44.6$ to $-14.7 \%)$ & 0.00031 \\
\hline & BPI & $-42.6 \%(-62.8$ to $-22.4 \%)$ & 0.00003 \\
\hline & $\mathrm{C} 5 \mathrm{a}$ & $-30.9 \%(-47.9$ to $-13.9 \%)$ & 0.00077 \\
\hline & $\mathrm{C} 9$ & $-22.3 \%(-32.7$ to $-12 \%)$ & 0.00010 \\
\hline & Cathepsin E & $-14.4 \%(-24.7$ to $-4 \%)$ & 0.00015 \\
\hline & CD177 & $-21.7 \%(-32.3$ to $-11.1 \%)$ & 0.00001 \\
\hline & $\mathrm{COX}-2$ & $-27.1 \%(-41.2$ to $-13 \%)$ & 0.00042 \\
\hline & C-reactive protein & $-33.8 \%(-48.5$ to $-19 \%)$ & 0.00005 \\
\hline & Cystatin F & $-27.5 \%(-60.4$ to $+5.4 \%)$ & 0.00034 \\
\hline & Haptoglobin & $-20.9 \%(-46.2$ to $+4.4 \%)$ & 0.00082 \\
\hline & HMGN1 & $-16.1 \%(-23.7$ to $-8.6 \%)$ & 0.00012 \\
\hline & IL-6 & $-14.8 \%(-25$ to $-4.6 \%)$ & 0.00040 \\
\hline & IL-16 & $-11.5 \%(-17.8$ to $-5.2 \%)$ & 0.00054 \\
\hline & MMP9 & $-29.7 \%(-48.8$ to $-10.6 \%)$ & 0.00095 \\
\hline & Myeloperoxidase & $-33.5 \%(-45.4$ to $-21.5 \%)$ & 0.00000 \\
\hline & Proteinase 3 & $-37.9 \%(-50.5$ to $-25.3 \%)$ & 0.00000 \\
\hline & Resistin & $-21.2 \%(-27.9$ to $-14.5 \%)$ & 0.00000 \\
\hline & S100A12 & $-27.7 \%(-42.1$ to $-13.4 \%)$ & 0.00041 \\
\hline & SAP & $-16.7 \%(-26.2$ to $-7.3 \%)$ & 0.00099 \\
\hline & SP-D & $-49.1 \%(-74.6$ to $-23.6 \%)$ & 0.00042 \\
\hline \multirow[t]{4}{*}{ Signaling/transport } & ACVR1B & $-11.2 \%(-17.2$ to $-5.1 \%)$ & 0.00067 \\
\hline & ASPGR1 & $-11.3 \%(-17.6$ to $-5 \%)$ & 0.00094 \\
\hline & KPNA2 & $-10.8 \%(-16.6$ to $-5.1 \%)$ & 0.00002 \\
\hline & PTK2 & $-10 \%(-22.7$ to $+2.7 \%)$ & 0.00072 \\
\hline Lipid & LOX-1 & $-32.7 \%(-48.9$ to $-16.4 \%)$ & 0.00026 \\
\hline Smooth muscle relaxation & PDE5A & $-14.9 \%(-20.9$ to $-8.8 \%)$ & 0.00003 \\
\hline \multicolumn{4}{|l|}{ Increased } \\
\hline Cell proliferation & REG4 & $+35.6 \%$ (5.6 to $65.6 \%)$ & 0.00046 \\
\hline \multirow[t]{3}{*}{ Metabolic } & ENPP7 & $+35 \%(8$ to $62 \%)$ & 0.00040 \\
\hline & hFABP & $+28.5 \%(11.9$ to $45 \%)$ & 0.00018 \\
\hline & GDF15 & $+23.7 \%$ (11.9 to $35.4 \%)$ & 0.00003 \\
\hline \multirow[t]{2}{*}{ Protein folding/transport } & PDI & $+36.2 \%(16.6$ to $55.8 \%)$ & 0.00000 \\
\hline & pIgR & $+57.2 \%(9.9$ to $104.5 \%)$ & 0.00054 \\
\hline Thrombosis & Protein $\mathrm{C}$ & $+10.3 \%(5.5$ to $15.2 \%)$ & 0.00013 \\
\hline Tissue repair & HGFA & $+9.9 \%(5.3$ to $14.5 \%)$ & 0.00011 \\
\hline
\end{tabular}

Mean percent change from baseline, adjusted for change in placebo.

$A 1 A C$ alpha 1-antichemotrypsin, BPI Bactericidal permeability-increasing protein, $C 5 a$ complement component $5 \mathrm{a}$, $C 9$ complement component 9 , CD177 cluster of differentiation 177, $C O X-2$ cyclooxygenase-2, HMGN1 high-mobility group nucleosome-binding protein 1 , IL interleukin, $M M P 9$ matrix metalloproteinase 9, S100A12 S100 calcium-binding protein A12, SAP serum amyloid P component, SP-D surfactant protein D, $A C V R 1 B$ activin receptor type-1B, ASPGR1 Asialoglycoprotein receptor 1, KPNA2 karyopherin alpha 2, PTK2 protein tyrosine kinase 2, LOX-1 oxidized low-density lipoprotein receptor 1, ENPP7 ectonucleotide Pyrophosphatase/Phosphodiesterase 7, PDE5A phosphodiesterase 5A, REG4 regenerating islet-derived protein $4, G D F 15$ growth/differentiation factor $15, h F A B P$ heart-type fatty acid binding protein, $P D I$ protein disulfideisomerase, $p I g R$ polymeric immunoglobulin receptor, $H G F A$ hepatocyte growth factor activator.

in insulin resistance in colchicine-treated subjects were also highly inversely correlated with changes in GDF15 levels. GDF15 is often released as part of a stress response after anoxia or tissue injury, although conflicting evidence exists whether it contributes to, or protects against, diabetes and CVD development. In large cohort studies, GDF15 
A

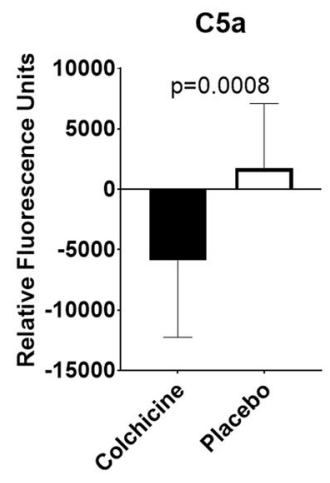

IL-6

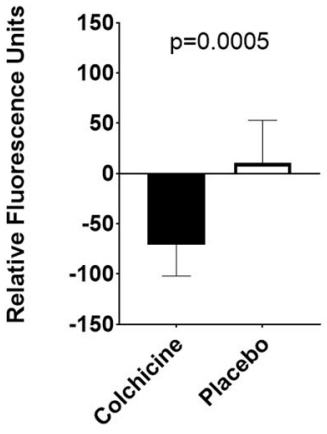

SAP

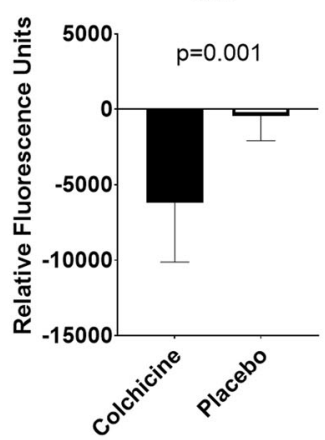

B

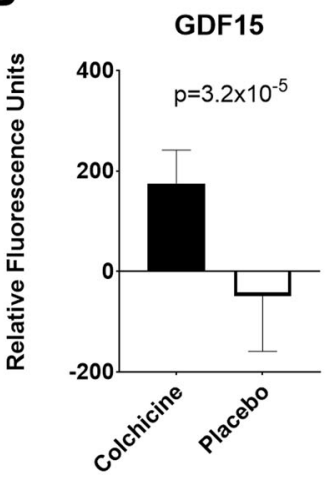

C9

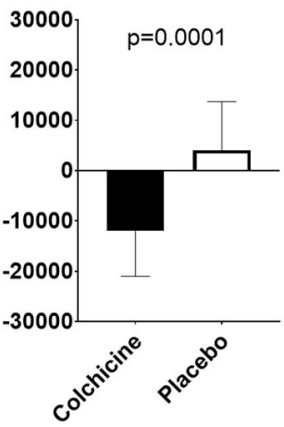

Myeloperoxidase

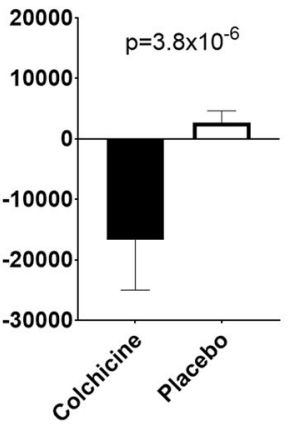

SF-D

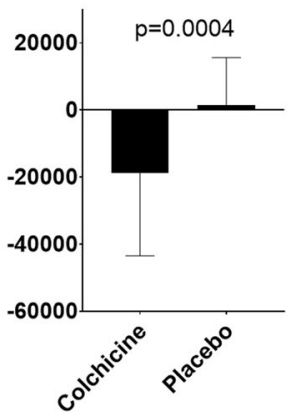

hFABP

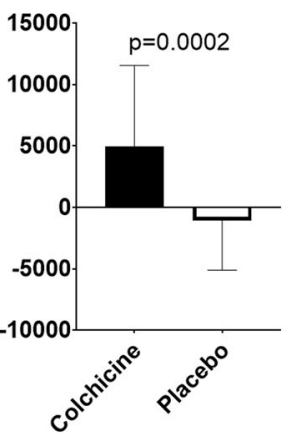

Fig. 1 Changes in Selected Circulating Biomarkers. Selected circulating biomarkers that were significantly $\mathbf{a}$ decreased or $\mathbf{b}$ increased in the colchicine arm as compared with placebo after adjustment for
COX-2

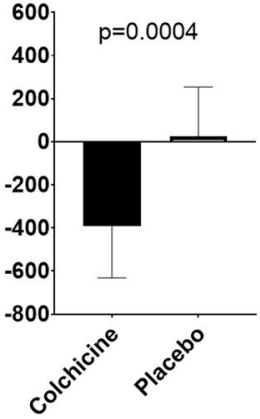

Proteinase 3

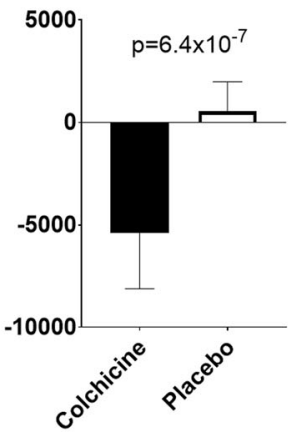

LOX-1

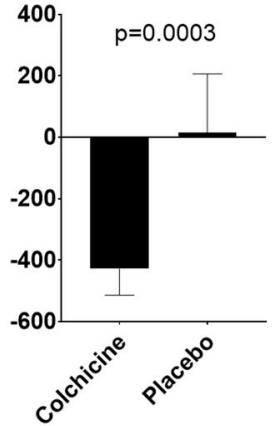

HGFA

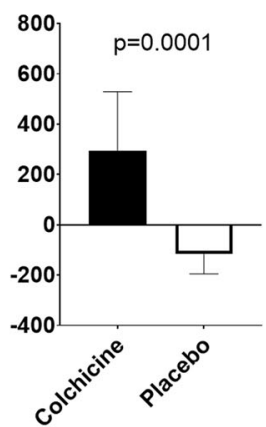

Haptoglobin

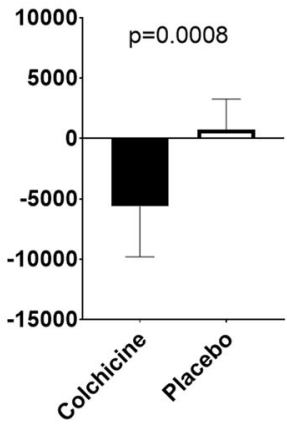

\section{Resistin}

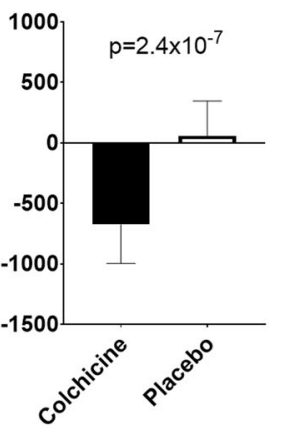

PDE5A

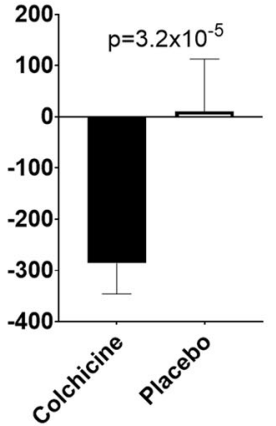

Protein C

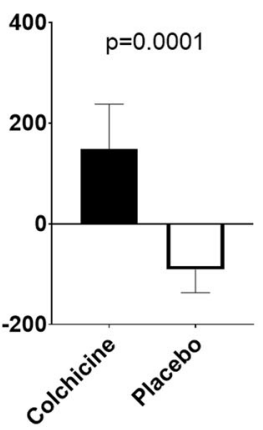

the false discovery rate. Bars represent median values, whiskers represent the $95 \%$ confidence interval. All values are expressed as relative fluorescence units. 
concentrations were associated with increased risk of diabetes, CVD, and mortality [12]. Conversely, in mouse models GDF15 was protective to ischemic cardiomyocytes [13] and injured pancreatic beta cells [14]. Interestingly, GDF15 may be responsible for metformin's beneficial effects on weight loss and energy expenditure [15], and colchicine's metabolic benefits may in part come from its ability to stimulate hepatic GDF15 production [16].

Resistin and LOX-1, two key regulators of immunometabolism, were significantly decreased by colchicine. In mouse models resistin is primarily secreted by adipocytes and has been shown to increase fasting blood glucose, insulin resistance, and hepatic glucose production [17]; in humans, resistin is primarily secreted by monocytes/macrophages and promotes vascular inflammation and atherogenesis. LOX-1, a receptor for oxLDL and regulator of cholesterol and fatty acid uptake in adipose and vascular tissue, can stimulate expression of adhesion molecules, inflammation, and promote atherosclerotic plaque formation [18]. We previously showed that colchicine paradoxically increased oxLDL [9]; the results from this analysis suggest that this may be due to reduced levels of its scavenger receptor removing it from circulation. Despite the increased oxLDL levels, less may be entering vascular tissue, thereby preventing vascular inflammation and atherosclerosis. Surprisingly, changes in LOX-1 levels did not correlate with changes in oxLDL among colchicine-treated patients, although this finding may simply be due to a lack of power.

Multiple inflammatory pathways and mediators implicated in diabetes and/or CVD development were also suppressed by colchicine. Consistent with previous reports, colchicine suppressed molecules associated with neutrophil function and activation [19]. Interestingly, other members of the innate immune system were also suppressed, including members of the complement cascade, haptoglobin, and surfactant protein D [20]. IL-6, a cytokine known to both positively [21] and negatively [22, 23] influence glucose homeostasis, was also significantly decreased by colchicine. Somewhat surprisingly, reductions in IL-6 were nominally significantly associated with reductions, rather than improvements, in first-phase insulin secretion and insulin-independent glucose disposal, suggesting that IL-6 inhibition may not be responsible for colchicine's beneficial metabolic effects.

However, colchicine's suppressive effects on IL-6 in obesity raise the question of its potential as a treatment for COVID-19, a disease caused by a novel coronavirus (CoV), SARS-CoV-2. Individuals with severe obesity and/or T2D are at increased risk for infection, complications, and death from COVID-19 [24]. Because a large proportion of COVID-19 mortality is thought to be brought on by a dysregulated cytokine storm, intense research is currently underway examining the use of anti-inflammatory medications [25]. Recent case reports have supported the use of the anti-IL-6 inhibitor, tocilizumab [26], and clinical trials investigating the use of colchicine in COVID-19 are currently underway [27]. Of course, given colchicine's antiinflammatory effects, a fear could be that it increases the risk of severe infection. Rates of mild or severe infections were not found to be significantly increased in our primary RCT analysis [5], nor in other previous colchicine studies $[28,29]$; however, colchicine's effects in COVID-19 remain to be seen.

A strength of the study was its randomized, double-blind, placebo-controlled design. In addition, given the large number of molecules assessed, our analyses adjusted for FDR. A limitation of the study was the relatively small sample size, which prevented us from performing an informative cluster analysis, and which may also have limited the ability to detect differences between groups. Additionally, the results presented are from a secondary analysis of the trial. However, we confirmed several findings (e.g., reductions in CRP, IL-6, and neutrophil markers) described previously $[8,19]$. Another limitation is that our subjects were at-risk for T2D and CVD development rather than having known disease. It is possible that colchicine's effects on biomarkers and atherosclerotic pathways may vary with degree of T2D or CVD severity.

In conclusion, in otherwise healthy adults with obesity and MetS, colchicine use was associated with reduction in multiple inflammatory mediators and pathways. Interestingly, biomarkers linked to pathways implicated in CVD, including lipid circulation, endothelial dysfunction, nitric oxide production, and thrombosis were also significantly changed by colchicine. Further studies are warranted to investigate which of these pathways prominently play a role in colchicine's metabolic and cardioprotective effects.

Supplementary information is available at the International Journal of Obesity's website.

Acknowledgements The authors would like to thank the NIH Clinical Center nurses of 5NW, the Metabolic Unit, and OP9 for their dedication and assistance in the carrying out of the clinical study.

CHI Consortium Huizhi Zhou ${ }^{4}$, Rongye $\mathrm{Shi}^{4}$, Poorani Subramanian ${ }^{4}$, John Tsang ${ }^{4}$, Yasmine Belkaid ${ }^{4}$

Funding 1ZIAHD000641, with supplemental funding from an NICHD Division of Intramural Research Director's Award. This research was also supported by the Intramural Research Program of the $\mathrm{NIH}$, National Institute of Allergy and Infectious Diseases, and the trans-NIH Center for Human Immunology.

\section{Compliance with ethical standards}

Conflict of interest The authors have no conflict of interest to disclose. JAY receives grant support for unrelated studies sponsored by Rhythm Pharmaceuticals Inc., and by Soleno Therapeutics Inc. 
Publisher's note Springer Nature remains neutral with regard to jurisdictional claims in published maps and institutional affiliations.

\section{References}

1. Wilson PW, D'Agostino RB, Parise H, Sullivan L, Meigs JB. Metabolic syndrome as a precursor of cardiovascular disease and type 2 diabetes mellitus. Circulation. 2005;112:3066-72.

2. Sattar N, Gaw A, Scherbakova O, Ford I, O'Reilly DS, Haffner $\mathrm{SM}$, et al. Metabolic syndrome with and without C-reactive protein as a predictor of coronary heart disease and diabetes in the West of Scotland Coronary Prevention Study. Circulation. 2003;108:414-9.

3. Nishida M, Moriyama T, Ishii K, Takashima S, Yoshizaki K, Sugita Y, et al. Effects of IL-6, adiponectin, CRP and metabolic syndrome on subclinical atherosclerosis. Clin Chim Acta. 2007;384:99-104.

4. Saklayen MG. The global epidemic of the metabolic syndrome. Curr Hypertens Rep. 2018;20:12.

5. Demidowich AP, Levine JA, Onyekaba GI, Khan SM, Chen KY, Brady SM, et al. Effects of colchicine in adults with metabolic syndrome: a pilot randomized controlled trial. Diabetes Obesity Metab. 2019;21:1642-51.

6. Wang L, Sawhney M, Zhao Y, Carpio GR, Fonseca V, Shi L. Association between colchicine and risk of diabetes among the veterans affairs population with gout. Clin Ther. 2015;37:1206-15.

7. Thompson PL, Nidorf SM. Colchicine: an affordable antiinflammatory agent for atherosclerosis. Curr Opin Lipidol. 2018;29:467-73.

8. Martinez GJ, Robertson S, Barraclough J, Xia Q, Mallat Z, Bursill $\mathrm{C}$, et al. Colchicine acutely suppresses local cardiac production of inflammatory cytokines in patients with an acute coronary syndrome. J Am Heart Assoc. 2015;4:e002128.

9. Demidowich AP, Wolska A, Wilson SR, Levine JA, Sorokin AV, Brady SM, et al. Colchicine's effects on lipoprotein particle concentrations in adults with metabolic syndrome: a secondary analysis of a randomized controlled trial. $\mathrm{J}$ Clin Lipidol. 2019;13:1016-22.

10. Rohloff JC, Gelinas AD, Jarvis TC, Ochsner UA, Schneider DJ, Gold L, et al. Nucleic acid ligands with protein-like side chains: modified aptamers and their use as diagnostic and therapeutic agents. Mol Ther Nucleic Acids. 2014;3:e201.

11. Cheung F, Fantoni G, Conner M, Sellers BA, Kotliarov Y, Candia $\mathrm{J}$, et al. Web tool for navigating and plotting SomaLogic ADAT files. J Open Res Softw. 2017;20:5.

12. Adela R, Banerjee SK. GDF-15 as a target and biomarker for diabetes and cardiovascular diseases: a translational prospective. J Diabetes Res. 2015;2015:490842.

13. Kempf T, Eden M, Strelau J, Naguib M, Willenbockel C, Tongers $\mathrm{J}$, et al. The transforming growth factor-beta superfamily member growth-differentiation factor-15 protects the heart from ischemia/ reperfusion injury. Circ Res. 2006;98:351-60.
14. Nakayasu ES, Syed F, Tersey SA, Gritsenko MA, Mitchell HD, Chan CY, et al. Comprehensive proteomics analysis of stressed human islets identifies GDF15 as a target for type 1 diabetes intervention. Cell Metab. 2020;31:363-74.

15. Coll AP, Chen M, Taskar P, Rimmington D, Patel S, Tadross JA, et al. GDF15 mediates the effects of metformin on body weight and energy balance. Nature. 2020;578:444-8.

16. Weng J, Koch P, Shimada T, Mitchison TJ. Hepatokine induction by colchicine prevents systemic inflammation via activating PTPN6 inhibitory signaling. In: ASCB Annual Meeting, Washington, DC. Mol Biol Cell 2019; 30: M184: Mol Biol Cell.

17. Steppan CM, Bailey ST, Bhat S, Brown EJ, Banerjee RR, Wright $\mathrm{CM}$, et al. The hormone resistin links obesity to diabetes. Nature. 2001;409:307-12.

18. Yan M, Mehta JL, Hu C. LOX-1 and obesity. Cardiovasc Drugs Ther. 2011;25:469-76.

19. Leung YY, Yao Hui LL, Kraus VB. Colchicine-Update on mechanisms of action and therapeutic uses. Semin Arthritis and Rheum. 2015;45:341-50.

20. Fernandez-Real JM, Valdes S, Manco M, Chico B, Botas P, Campo A, et al. Surfactant protein d, a marker of lung innate immunity, is positively associated with insulin sensitivity. Diabetes Care. 2010;33:847-53.

21. Pedersen BK. IL-6 signalling in exercise and disease. Biochem Soc Trans. 2007;35:1295-7.

22. Klover PJ, Zimmers TA, Koniaris LG, Mooney RA. Chronic exposure to interleukin- 6 causes hepatic insulin resistance in mice. Diabetes. 2003;52:2784-9.

23. Rotter V, Nagaev I, Smith U. Interleukin-6 (IL-6) induces insulin resistance in 3T3-L1 adipocytes and is, like IL-8 and tumor necrosis factor-alpha, overexpressed in human fat cells from insulin-resistant subjects. J Biol Chem. 2003;278:45777-84.

24. Muniyappa R, Gubbi S. COVID-19 pandemic, corona viruses, and diabetes mellitus. Am J Physiol Endocrinol Metab. 2020;318: E736-41.

25. Mehta P, McAuley DF, Brown M, Sanchez E, Tattersall RS, Manson JJ, et al. COVID-19: consider cytokine storm syndromes and immunosuppression. Lancet. 2020;395:1033-4.

26. Michot JM, Albiges L, Chaput N, Saada V, Pommeret F, Griscelli F, et al. Tocilizumab, an anti-IL6 receptor antibody, to treat Covid-19-related respiratory failure: a case report. Ann Oncol. 2020.

27. Deftereos SG, Siasos G, Giannopoulos G, Vrachatis DA, Angelidis C, Giotaki SG, et al. The GReek study in the Effects of Colchicine in COvid-19 complications prevention (GRECCO19 study): rationale and study design. Hellenic J Cardiol. 2020.

28. Tardif JC, Kouz S, Waters DD, Bertrand OF, Diaz R, Maggioni AP, et al. Efficacy and safety of low-dose colchicine after myocardial infarction. N Engl J Med. 2019;381:2497-505.

29. Nidorf SM, Eikelboom JW, Budgeon CA, Thompson PL. Lowdose colchicine for secondary prevention of cardiovascular disease. J Am Coll Cardiol. 2013;61:404-10. 